\title{
New light on the formation and evolution of bars
}

\section{Trends in the stellar line-strength indices distribution inside the bar region $\star$}

\author{
I. Pérez ${ }^{1, \star \star, 2, \star \star \star}$, P. Sánchez-Blázquez $z^{3, \dagger}$, and A. Zurita ${ }^{2, \ddagger}$ \\ 1 Kapteyn Astronomical Institute, University of Groningen, The Netherlands \\ e-mail: isa@astro.rug.nl \\ 2 Departamento de Física Teórica y del Cosmos, Universidad de Granada, Spain \\ e-mail: azurita@ugr.es \\ 3 Centre For Astrophysics, University of Central Lancashire, UK \\ e-mail: psanchez-blazquez@uclan.ac.uk
}

Received 6 December 2006 / Accepted 31 January 2007

\section{ABSTRACT}

\begin{abstract}
Aims. Our aim is to study the stellar content of the bar region to constrain its formation and evolution. Methods. Line-strength indices in the bar region of a sample of 6 barred galaxies were employed to derive age and metallicity gradients along the bars using stellar population models.

Results. We find clear radial gradients in the line-strength indices for all the galaxies. We find positive gradients within the bar region in the metal indices in four of the six galaxies and opposite trends in the other two. These two galaxies are classified as SAB, and they present exponential bar light profiles. For all the galaxies, we find a positive gradient in the Balmer indices. There is a clear correlation between the position of morphological features inside the bar region with changes in the slope and value of the indices, which indicate changes in the stellar populations, when using stellar population analysis. Therefore, it seems that the bar regions show a gradient in both age and metallicity, changing radially to younger and more meta-rich populations for all the galaxies except for the two with exponential profiles.
\end{abstract}

Key words. galaxies: abundances - galaxies: formation - galaxies: evolution

\section{Introduction}

The importance of bars in galaxies as mechanisms for transfering angular momentum and matter on large scales within the galaxy is indisputable (Kormendy \& Kennicutt 2004, and references therein). Bars are thought to be formed through spontaneous disk instabilities or by instabilities produced during galaxy encounters; the details of bar formation and their subsequent evolution remains a matter of debate. Until now, detailed analyses of the gas-phase abundances along bars have only been undertaken from observations of the emission lines from HII regions. Studies of the gas-phase abundances provide us with a current snapshot of the interstellar medium abundance. These studies have shown that there is little variation in the chemical abundances in the gas phase along the bar (Martin \& Friedli 1997,1999). Study of the stellar ages and metallicities, on the other hand, gives us "archaeological" clues as to the formation and evolution of the bar. Furthermore, the evolution of the two components, gas and stars, suffers from very different evolutionary processes; the gas is mainly dominated by the gravitational torque of the non-axisymmetric mass component, while the

* Based on observations obtained at Siding Spring Observatory (RSAA, ANU, Australia)

$\star \star$ Veni Fellow.

$\star \star \star$ Associate Researcher.

Marie Curie.

Retorno J.A Fellow. evolution of the stellar component is mainly affected by different orbital mixing (Binney \& Tremaine 1987), so one would not expect the same abundance trends in both components. Friedli (1998) used $N$-body simulations of bars with pre-existing exponential abundances to predict a null evolution of the stellar abundance profile (although with a decrease in the mean metallicity) while the gas abundance profile flattened rapidly. As mentioned before, the gas-phase abundance distribution predicted by Friedli (1998) has been shown to agree with the observational data of abundances in HII regions. There has not been any study addressing the stellar population along bars using spectroscopic information. Recently, Moorthy \& Holtzman (2006) have presented results for the stellar populations of the bulges of spiral galaxies, some of which are barred galaxies, finding a difference between bulges of barred and unbarred galaxies.

To date, it has been difficult to obtain radial abundance distributions along bars because, in order to obtain reliable stellar abundances and ages, very high signal-to-noise $(\mathrm{S} / \mathrm{N})$ spectra are necessary, and although bars are high surface brightness structures, this still implies long integration times on mediumsize telescopes. We carried out a project to obtain high $\mathrm{S} / \mathrm{N}$ data along the bar major axis of a sample of barred galaxies. This is the first detailed study of stellar line indices within the bar region. In this letter, we show clear evidence of line-strength index gradients along the bars and a correlation between the position of morphological features inside the bar region (bulge/innerdisk/inner-bar/primary-bar) and breaks in their respective stellar populations. 


\section{Observations and data reduction}

We selected barred galaxies from the Third Reference Catalogue of bright galaxies (RC3) (de Vaucouleurs 1948) with the following criteria; to be southern $\left(\delta<0^{\circ}\right)$, classified as barred and with inclinations between $10^{\circ}$ and $50^{\circ}$, and to be nearby $\left(c z \leq 2500 \mathrm{~km} \mathrm{~s}^{-1}\right)$ to properly resolve the bar, with morphological types earlier than SBb to avoid any morphological dependency on the results (in this first approach) and to ensure that the requested $\mathrm{S} / \mathrm{N}$ is reached, since early-type galaxies have higher bar surface brightness, crucial for the line-strength determination. We finally observed six galaxies (names and morphological types are indicated in Fig. 1). It is by no means a statistically complete sample, but it is a starting point in the study of the line-strength indices in bars.

We obtained long-slit spectra of six barred galaxies with the Double Beam Spectrograph (DBS) on the $2.3 \mathrm{~m}$ telescope at Siding Spring Observatory (SSO) during February 2006. SITe $1752 \times 532$ CCD detectors were used. The gratings employed were the $600 \mathrm{~B}$ and the $600 \mathrm{R}$ for the blue and red arms, respectively, with a slit-width of 2 arcsec. This set-up gives a dispersion of $1.1 \AA$ /pixel for the blue arm and $1.09 \AA /$ pixel for the red arm in the wavelength intervals 3892-5814 $\AA$ and 5390-7314 $\AA$, respectively, giving a velocity dispersion resolution $\Delta \sigma \approx 130 \mathrm{~km} \mathrm{~s}^{-1}$. The slit was placed along the bar major axis. The slit length is 7 arcmin, which allows one to study nearby galaxies in detail, while still providing sufficient sky coverage. The position angle was derived using the DSS images of the sample galaxies. Comparison arc-lamp exposures were obtained for wavelength calibration. Spectrophotometric standards were observed with a slit width of 6 arcsec. Additionally, we observed $11 \mathrm{G}-\mathrm{K}$ stars from the Lick/IDS library to be used as templates for velocity dispersion $(\sigma)$ measurements, as well as to transform our line-strength indices to the Lick system. The total integration time for each of the galaxies was typically 3 hours.

The data presented in this letter refers to the blue range alone. All the spectra were reduced using standard IRAF ${ }^{1}$ routines. Overscan and bias were subtracted from all the frames. No dark subtraction was done due to the low dark current of the chips used. Flatfielding correction was achieved to the $2 \%$ level. The relatively small size of the galaxies, compared to the slit length, allowed for accurate sky subtraction for each frame prior to combining the reduced spectra. A first-order polynomial was fit along the spatial direction and then subtracted from the frames. Spectrophotometric standards were used to relatively flux-calibrate the spectra. For each fully reduced galaxy frame, a final frame was created by extracting spectra along the slit, binning in the spatial direction to guarantee a minimum $\mathrm{S} / \mathrm{N}$ of 20 per $\AA$ in the spectral region of $\mathrm{Mgb}$.

\section{Line-strength indices}

In order to analyse the stellar population of the bars, spectral indices of the Lick/IDS system (Faber et al. 1985) were measured and are presented in Fig. 1. We adopted the definition of Trager et al. (1998) and Worthey \& Ottaviani (1997) for the high-order Balmer lines $\mathrm{H} \delta$ and $\mathrm{H} \gamma$. The $\mathrm{Fe} 3 \mathrm{n}$ index derived here is a combination of three prominent Fe lines

1 IRAF is distributed by the National Optical Astronomy Observatories, which is operated by the Association of Universities for Research in Astronomy, Inc. (AURA) under cooperative agreement with the National Science Foundation.
$(\mathrm{Fe} 4383+\mathrm{Fe} 5270+\mathrm{Fe} 5335) / 3)$, similar to the Fe3 index defined by Kuntschner (2000), with Fe4383 instead of Fe5015.

To compare between different regions of the galaxy and to compare with other galaxies, indices have to be measured at the same resolution. To allow flexibility in the model selection and to potentially compare with the measurements of other galaxy components from other studies, we decided to follow this approach. After broadening to the Lick resolution, line-strength indices were measured using the INDEX routine (Cardiel 1999).

To derive errors in the line-strength indices, we followed a similar approach to the one in Kelson et al. (2006). This method consists in calculating the errors in the mean flux within a passband using noise spectra derived by subtracting a bestfit model to the galaxy spectra. To derive the best-fit we used the OPTEMA algorithm described by González (1993).We used the synthetic spectral energy distributions provided by Vazdekis et al. (2007). Once the error in the mean flux of each bandpass is calculated, the formulae derived in Cardiel et al. (1998) were used to calculate the errors in the indices. As the values of the indices depend on the Doppler broadening, the velocity dispersion along the radii was also calculated. To derive $\sigma$ and radial velocities, we used the MOVEL and OPTEMA algorithms described by González (1993). In order to build an optimal template, we used stars observed with exactly the same instrumental configuration as the galaxies. The correction of the indices for the velocity dispersion broadening was done following the standard procedure of deriving correction curves from artificially broadened spectra. We derived a different curve for each galaxy using the best-fit spectra obtained in the derivation of the errors. The reason for deriving a polynomial for each galaxy is that the broadening correction depends on the strength of the indices (Kuntschner 1998) and by adopting a single polynomial for all the galaxies, artificial trends between indices and $\sigma$ can be introduced (Kelson et al. 2006).

To correct the indices for any nebular emission contribution, we used GANDALF (Sarzi et al. 2006) which is a simultaneous emission and absorption-line fitting algorithm. We marked all the points with detected emission (see Fig. 1), where the detection threshold was calculated with the prescriptions given in Sarzi et al. (2006). The sensitivity varies from $0.3 \AA$ in the internal parts to $0.6 \AA$ in the external parts of the galaxy. When the emission completely fills all the absorption Balmer lines, the correction is more uncertain. For those cases, we used different symbols in Fig. 1.

We used TMB03, Bruzual \& Charlot (2003), and Vazdekis et al. (2007) models to derive simple stellar population ages and metallicities with the indices $\mathrm{H} \beta$ and $[\mathrm{MgbFe}]^{\prime}$ (TMB03). We also derived $[\alpha / \mathrm{Fe}]$ using $\mathrm{Fe} 4383$ and Mgb by fixing the ages obtained in the $[\mathrm{MgbF}]^{\prime}$ vs. $\mathrm{H} \beta$ diagram and the TMB03 models.

\section{Location of the morphological features}

In order to locate the radius of changes in morphological structure, ellipse fitting to the light distribution was performed with the IRAF task ELLIPSE. All the galaxies showed point-like nuclear regions, so the centre was fixed using the coordinates obtained by fitting a Gaussian to the nucleus. The position angle (PA) and the ellipticity (e) were left as free parameters in the fitting. Changes in both ellipticity and PA were considered as candidate structures, and visual inspection of the images was then performed to see if there was any anomaly. This analysis is similar to that described in Erwin (2004). The images used for the analysis come from different sources, all the analysis was 

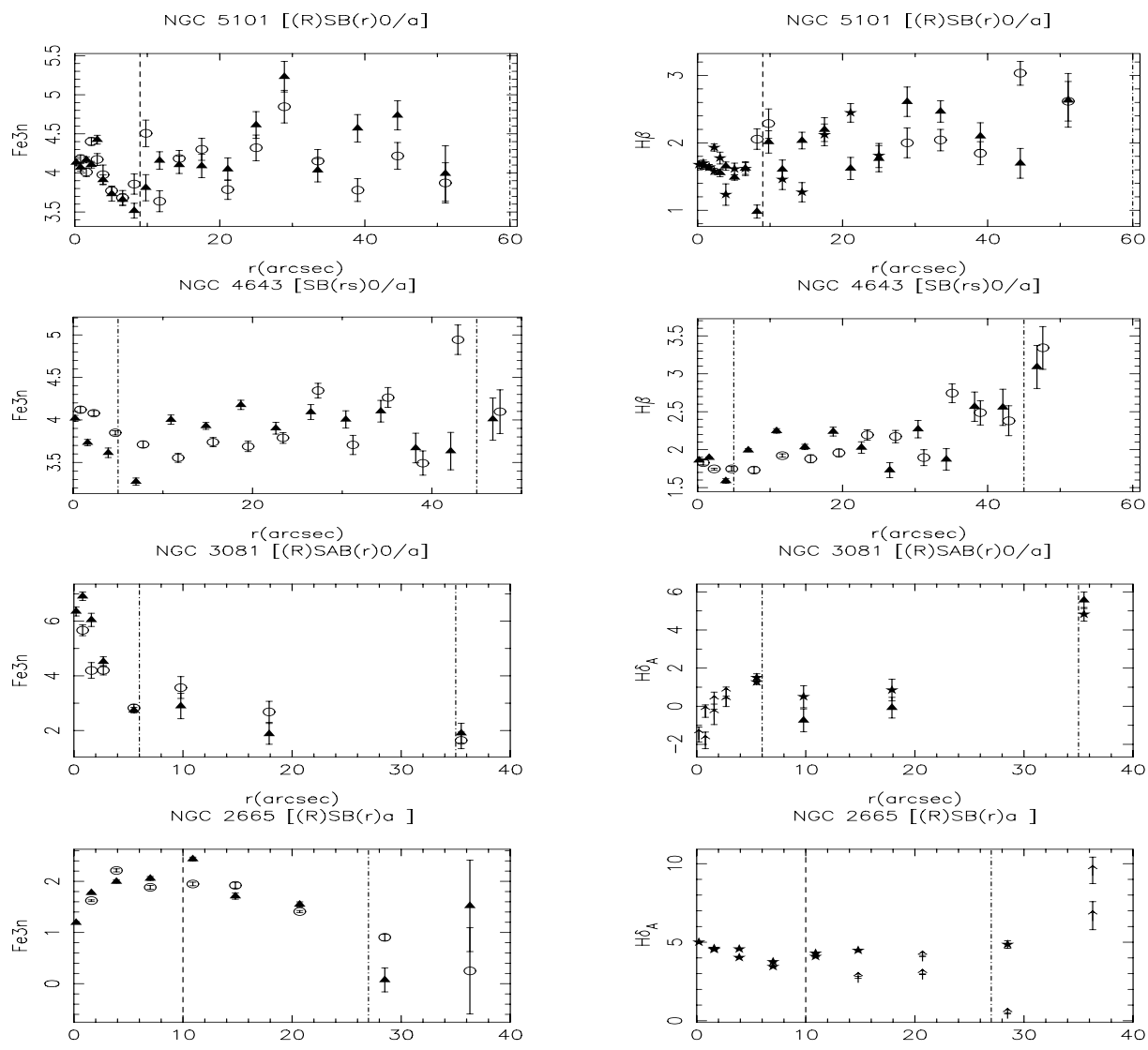

NGC $2217[(\operatorname{Rrcsec}) \operatorname{sB}(r s) 0 / a]$
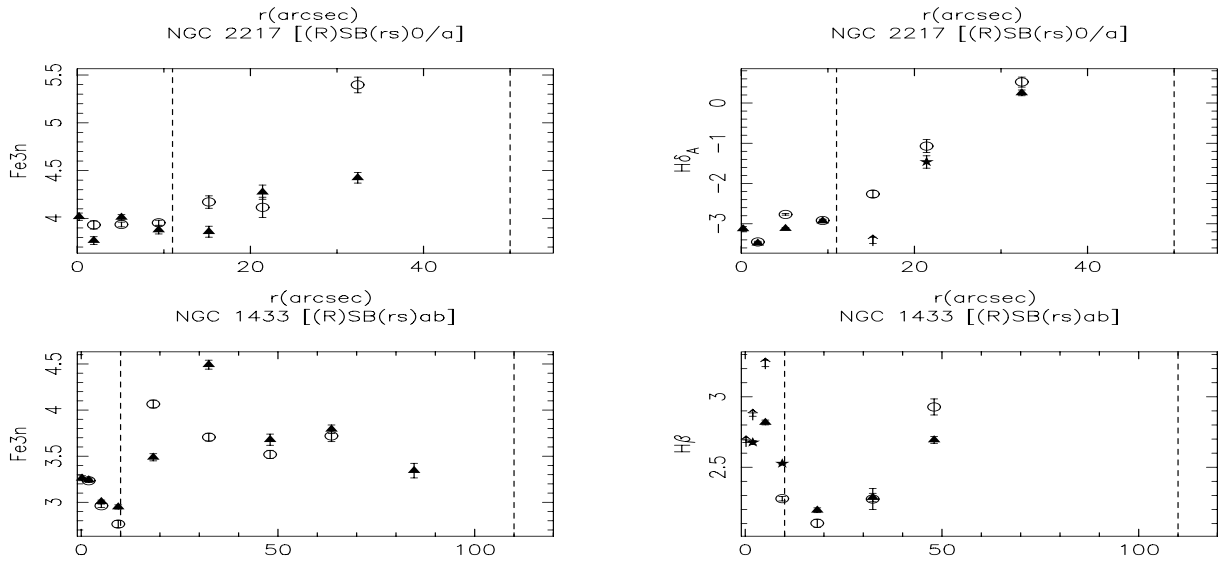

Fig. 1. Radial distribution of two indices for each of the galaxies (a combined $\mathrm{Fe}$ index and the $\mathrm{H}_{\beta}$ index or the $\mathrm{H}_{\delta}$ image depending on the emission in the bin, see text). The open circles and the filled triangles represent both sides of the bar. We have marked all the points with detected emission with a star, where the detection threshold has been calculated with the prescriptions given in Sarzi et al. (2006). When the emission completely fills all the absorption Balmer lines, the correction is more uncertain. For thoses cases, we have marked the points with an arrow. The vertical dashed lines represent the end-point of morphological features, meaning the inner bar or nuclear disk and the primary bar. Notice the general change in the indices for the two regions and the gradients in both.

done with 2MASS data and also a different band in the cases were there were published images. The following sources were used for each of the galaxies: the HST image of the nuclear region in the $F 606 W$ band for NGC 3081 (Malkan et al. 1998), the $R$-band by Hameed \& Devereux (1999) for NGC 1433, and the $B$-band image by Eskridge et al. (1999) for NGC 5101. We compared the results in the different bands and found good agreement in the position of the morphological features.

For three out of the six galaxies (NGC 1433, NGC 3081, and NGC 2217), there are published data on the radii of morphological structures within the inner region (Erwin 2004). There is good agreement (differences of $\approx 5 \%$ ) between our analysis and the values found by Erwin (2004).

\section{Results and summary}

Figure 1 shows the radial trends of two indices for each of the galaxies, a metal index and a Balmer one. The other Balmer indices follow the same trends as shown in Fig. 1. The Mg indices in some cases differ from the Fe indices, following opposite trends. However, one has to bear in mind that raw line indices are affected by the age-metallicity degeneracy. Therefore, 
disentangling the age and metallicity effects from the indices requires the use of stellar population models. In the cases where $\mathrm{H} \beta$ is more affected by emission, $\mathrm{H} \delta$ was chosen, since it is less affected by the emission lines (see Sect. 3 about emission correction). One should be careful when deriving the Balmer indices affected with emission since the derived values are sensitive to the emission correction (increasing the value after the correction). We have tried several methods (even no correction) and the trends do not change. In Fig. 1 one can clearly see a different trend in the radial distribution of the indices with a change at the radius of the inner structure (in Fig. 1, the innermost vertical line).

For clarity, we divide the results of the radial distribution in: a) the inner structure up to the innermost vertical line in Fig. 1, and $b$ ) the bar region between the two vertical lines in Fig. 1.

a) Inner region. There seems to be a gradient of the metal and Balmer lines within the inner structure, without taking the central points into account. These very central points are possibly dominated by the bulge and show a different behaviour for the bar and the inner structure. Both sides of the bar show similar trends, reinforcing the results.

b) Bar region. Within the bar region the slope changes for both the metal and the Balmer indices. For NGC 5101 and NGC 4643, there seems to be a point where the slope changes again corresponding to the radius around the point of maximum ellipticity, which is not considered as the end of the bar in the ellipticity-PA analysis. NGC 1433 shows an $\mathrm{Fe} 3 \mathrm{n}$ distribution that is also compatible with no radial change in the $\mathrm{Fe} 3 \mathrm{n}$, but there is a clear difference between the inner part and the bar region. The Balmer indices show a positive gradient and a change compared to the inner region. For two cases (NGC 2665 and NCG 3081) there seems to be a negative trend in the metal indices and a slightly negative trend in the Balmer indices; they do not show a change in the slope in the bar region. Interestingly enough, these are the only two galaxies classified as SAB in the sample, and they present an exponential profile in the bar, which might indicate a different formation process and history compared to those with a flat profile (Combes \& Elmegreen 1993; Noguchi 1996). This point deserves further investigation. For all galaxies, there is also good agreement between both sides of the bar.

There is an obvious change in the radial distribution of the different morphological components. We made tests by subtracting a bulge component (from the decomposition of the light profile), this only helps to obtain an even clearer result for the bar and inner structure, without changing the basic trends. We prefer to show the complete radial profile in order to avoid affecting the observed profiles with any model-dependent analysis.

Independent of the selected models (see Sect. 3), we derived positive age and metallicity gradients in the bars (outer parts of the bars being younger and more metal rich than the internal parts), except for the two galaxies with exponential bars (NGC 2665 and NGC 3081). In a future paper we plan to do a more detailed analysis of the star formation history and chemical evolution of all the objects. There, we will present the comparison of these results with dynamical and stellar population models using the entire spectra to try to interpret the results in the context of bar formation and evolution.

In summary, we have obtained high $\mathrm{S} / \mathrm{N}$ long-slit spectra along the bars of six early-type barred galaxies and derived radial profiles of the line-strength indices for the sample galaxies. There is a clear difference between the indices in the bar region (in Fig. 1, the region between the two vertical lines) and the central component. Within the bar region there is a clear gradient in both metal and Balmer lines that, when combined with stellar population models for all galaxies, gives a gradient in both metallicity and age, indicating that outer parts of the bar are younger and more metal rich. These results might indicate a secular inside-out growth for the bar.

Acknowledgements. We are grateful to Reynier Peletier for allowing us to compare the emission correction with his code. We are really grateful to B. Gibson and K. Ganda for the careful reading of the manuscript. We thank the referee, A. Vazdekis, for very useful discussions and comments on the manuscript. This publication made use of data products from the Two Micron All Sky Survey, which is a joint project of the University of Massachusetts and the Infrared Processing and Analysis Center/California Institute of Technology, funded by the National Aeronautics and Space Administration and the National Science. I.P. acknowledges financial support from the Netherlands Organisation for Scientific Research (NWO) Foundation and the Leids Kerkhoven-Bosscha Fonds. A. Z. acknowledges support from the Consejería de Educación y Ciencia de la Junta de Andalucía, Spain.

\section{References}

Binney, J., \& Tremaine, S. 1987, Galactic Dynamics (Princeton: Princeton Univ. Press)

Bruzual, G., \& Charlot, S. 2003, MNRAS, 344, 1000

Cardiel, N. 1999, Ph.D. Thesis

Cardiel, N., Gorgas, J., Cenarro, J., \& González, J. 1998, A\&AS, 127, 597

Combes, F., \& Elmegreen, B. 1993, A\&A, 271, 391

de Vaucouleurs, G. 1948, Ann. Astrophys., 11, 24

Erwin, P. 2004, MNRAS, 364, 283

Eskridge, P., Frogel, J., Pogge, R., et al. 1999, AJ, 118, 730

Faber, S. M., Friel, E. D., Burstein, D., \& Gaskell, C. M. 1985, ApJS, 57, 711

Friedli, D. 1998, ASP Conf. Ser., 147, 287

González, J. J. 1993, Ph.D. Thesis

Hameed, S., \& Devereux, N. 1999, AJ, 118, 730

Kelson, D. D., Illingworth, G. D., Franx, M., \& van Dokkum, P. G. 2006, ArXiv Astrophysics e-prints

Kormendy, J., \& Kennicutt, R. 2004, ARA\&A, 42, 603

Kuntschner, H. 1998, Ph.D. Thesis

Kuntschner, H. 2000, MNRAS, 315, 184

Malkan, M., Gorjian, V., \& Tam, R. 1998, ApJS, 117, 25

Martin, P., \& Friedli, D. 1997, A\&A, 326, 449

Martin, P., \& Friedli, D. 1999, A\&A, 346, 769

Moorthy, B., \& Holtzman, J. 2006, MNRAS, 371, 583

Noguchi, M. 1996, ApJ, 469, 605

Sarzi, M., Falcón-Barroso, J., Davies, L., et al. 2006, MNRAS, 366, 1151

Trager, S. C., Worthey, G., Faber, S. M., Burstein, D., \& Gonzalez, J. J. 1998, ApJS, 116, 1

Worthey, G., \& Ottaviani, D. L. 1997, ApJS, 111, 377 